\title{
Zwischen Akzeptanz und Ablehnung: Modifikationen im Orofazialbereich unter besonderer Berücksichtigung von Dental Piercing und Tattoos
}

Gereon Schäfer

\section{Einleitung}

Körpermodifikationen erfreuen sich seit einigen Jahren besonderer Beliebtheit. Diese Popularität korreliert mit der Aufmerksamkeit, die sie gerade in jüngster Zeit von Seiten der Medien, der Wissenschaft und der Künste erfahren: So sind unlängst mehrere, teilweise viel beachtete Veröffentlichungen zu diesem Themenbereich erschienen. ${ }^{1}$ Die Süddeutsche Zeitung brachte im Februar einen Beitrag mit der Überschrift „Das ist mein Fleisch - Gespaltene Zungen, durchbohrte Glieder, Tattoos als ewige Zeichen: Warum machen die Menschen das?“", und im April 2007 befasste sich eine Ausstellung ${ }^{3}$ mit dem Titel „Unter die Haut: Tattoo und Piercing in 20 Portraits" mit dem besagten Sujet.

Der vorliegende Beitrag greift das Thema Körpermodifikationen - oder neudeutsch BodMod - auf, fokussiert dabei jedoch einen Teilaspekt, nämlich Modifikationen im Bereich des Orofazialsystems. Auch dieses Gebiet ist zunehmend Gegenstand von wissenschaftlichen Vorträgen ${ }^{4}$ und Aufsätzen, ${ }^{5}$ wenngleich

1 Kasten (2006), Vandekerckhove (2006), Gugutzer (2006).

2 Kissler (2006).

3 Ausstellung von Aglaja Stirn und Oli Hege vom 01.02. bis 15.04.2007 im Museum für Kommunikation in Frankfurt am Main.

$4 \operatorname{Groß}(2007 a)$.

5 Maio (2006). 
die betreffenden Beiträge zumeist einzelne Teilbereiche wie Dental Bleaching, Dental Piercing oder Tätowierungen in den Blick nehmen.

Ziel dieses Aufsatzes ist es, die Vielzahl der im Orofazialbereich nachweisbaren Körpermodifikationen darzustellen, zu differenzieren und einer näheren Betrachtung zu unterziehen. Dabei soll zunächst - ausgehend von einem historischen und ethnologischen Rückblick (Kapitel 2) - versucht werden, eine aktuelle und möglichst vollständige Übersicht über intentionale Modifikationen im Bereich des Orofazialsystems zu geben (Kapitel 3). Kapitel 4 beschäftigt sich unter dem Titel „Zwischen Selbstbestimmung und Selbstverletzung“ mit den Motiven der Träger von Körpermodifikationen, aber auch mit den Risiken der besagten Eingriffe. Im Anschluss daran wird die Frage aufgeworfen, inwieweit Träger von Piercings und Tattoos mit gesellschaftlicher Diskriminierung und Stigmatisierung rechnen müssen (Kapitel 5). Am Ende steht ein kurz gefasster Ausblick auf künftige Entwicklungen im Bereich der Körpermodifikationen (Kapitel 6).

\section{Historischer und ethnologischer Rückblick auf Körpermodifikationen im Orofazialbereich}

Körpermodifikationen, insbesondere permanenter Hautschmuck in Form von Tätowierungen oder Piercings, sind vermutlich so alt wie die Menschheit selbst. Sie wurden und werden in unterschiedlichen Epochen und Kulturen entwickelt und durchgeführt. Obgleich Tätowierungen und Piercings im westlichen Kulturkreis zumindest bis zur jüngsten Vergangenheit nur wenig gesellschaftliche Akzeptanz fanden, belegt doch z. B. der Fund der etwa 5.300 Jahre alten Eismumie in den Schnalstaler Gletschern (Südtirol), dass das Tätowieren der Haut bereits zu jener Zeit in Europa bekannt war. ${ }^{6}$ Auch das Piercen der Brustwarzen war schon bei den Zenturionen des Römischen Reiches üblich. ${ }^{7}$

Auch Körpermodifikationen im orofazialen Bereich, darunter eingreifende Veränderungen im Bereich der Zähne, sind in vielen Kulturen der Welt, vor allem in Südost-Asien, Mittel- und Südamerika sowie in Zentralafrika, verankert und teilweise bis zum heutigen Tage weit verbreitet. Dabei sind verschiedene Formen der Körpermodifikation zu unterscheiden:

Zum ersten sind Tätowierungen im Fazialbereich zu nennen: Sie können Teil von Tätowierungen sein, welche den Körper von Kopf bis Fuß überziehen, wie bei den Mentawaiern in Indonesien. ${ }^{8}$ Andere Stammesgesellschaften wie die Maori auf Neuseeland kennzeichnen ihre soziale Stellung durch besonders aufwändige Tätowierungen speziell des Gesichts. ${ }^{9}$

Zum zweiten lassen sich Piercings im Orofazialbereich anführen: Bei Eskimostämmen in Alaska etwa werden den Jungen als zeremonielles Zeichen ihres Eintritts in die Pubertät die Unterlippen im Bereich der Mittellinie durch-

6 www.archaelogiemuseum.it/f01_de.htm [07.03.2007].

7 Armstrong et al. (1995).

8 Zahorka (2001).

9 Vandekerckhove (2006), Abbildung S. 17. 
stoßen und in die Perforationen Objekte aus Holz oder Knochen eingesetzt. ${ }^{10}$ Noch weiter gehende Lippenveränderungen zählen zum Brauchtum einiger Nomadenstämme im Südwesten Äthiopiens: So wird bei jungen Frauen vom Stamme der Mursi zu Beginn ihrer Pubertät die Unterlippe mittig durchbohrt und anschließend durch Einlegen von verschiedenen Tontellern zunehmender Größe auf einen Durchmesser von durchschnittlich sechs bis sieben Zentimetern gedehnt (s. Abb. 1). Zusätzlich müssen die beiden zentralen Schneidezähne des Unterkiefers geopfert werden, um der mit einfachen Motiven geschmückten Scheibe über diese Zahnlücke eine horizontale Abstützung zu vermitteln. Auf diese Weise wird gewährleistet, dass durch das Gewicht der Tonscheibe die Lippe nicht nach unten gezogen wird. ${ }^{11}$

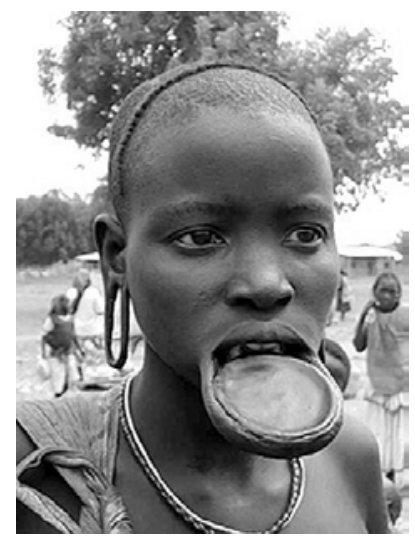

Abb. 1 Junge Mursi-Frau mit Lippenschmuck

Bei den Surmi, einem Stamm aus der gleichen Region Äthiopiens, werden von den Frauen bis zu zehn Zentimeter große Holzplatten in der Unterlippe getragen (s. Abb. 2). ${ }^{12}$

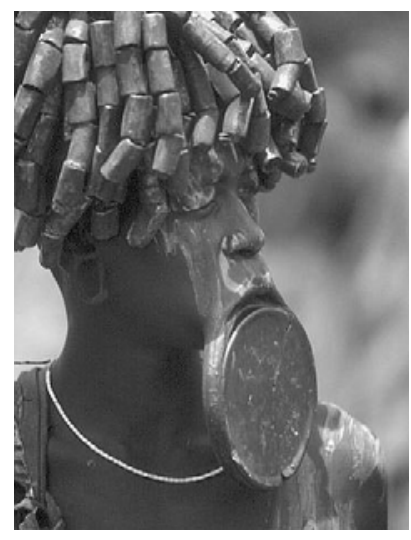

Abb. 2 Surmi-Frau mit Lippenschmuck

10 Aufderheide/Rodríges-Martín (1998).

11 Zahorka (2001).

12 Aufderheide/Rodríges-Martín (1998), Zahorka (2001), Scully/Chen (1994). 
Völker wie die Dogon aus Mali oder die Toposa aus dem Sudan tragen in Unter- oder Oberlippe Pflöcke aus Holz. ${ }^{13}$ Ihren Familienstand kennzeichnen die Frauen der Toposa, indem sie einen Metalldraht in der Mitte ihrer Unterlippe fixieren. ${ }^{14}$ Bei einer auch heute noch stattfindenden religiösen Zeremonie in Malaysia und Südthailand, dem Thaipusam-Festival, werden hauptsächlich die Wangen, aber auch Zunge und Lippen mit verschiedensten Gegenständen durchstoßen oder durchbohrt. ${ }^{15}$ Ebenfalls spirituell motiviert waren Zungenpiercings bei den Maya. ${ }^{16}$

Hiervon abzugrenzen sind drittens dentale Modifikationen. Die traditionellen Eingriffe im Bereich der Zähne können nach Schröder $(1906)^{17}$ in sieben Kategorien eingeteilt werden:

\section{Einfache Zuspitzung der Zähne}

Das Anspitzen der Zähne ist die häufigste und am weitesten verbreitete Form traditioneller Zahnveränderungen. Entsprechend vielfältig stellen sich die Motive dar: So erhofft sich der Stamm der Niam-Niam in Zentralafrika eine Verbesserung der Effizienz des Gebisses bei der Nutzung als Waffe im Kampf gegen den Feind. ${ }^{18}$

Andere Völker oder Stämme wünschen sich Zähne, die das Aussehen von bestimmten Tieren haben („scharf wie Mäusezähne“, „scharf wie Haifischzähne“, „aussehen wie ein Raubtier“"). ${ }^{19}$

Ein besonders eindrucksvolles Beispiel für eine kulturell motivierte Mutilation der Zähne liefern die indigenen Bewohner der indonesischen MentawaiInseln: Meist schon vor Eintritt in die Pubertät werden die Schneidezähne der Jungen und Mädchen in Ober- und Unterkiefer durch Schläge mit einem meißelartigen Werkzeug dreieckig zugespitzt (s. Abb. 3). Diese sehr schmerzhafte Prozedur dauert nicht länger als eine halbe Stunde und hat ihre Wurzeln in einer etwa 5.00o Jahre alten neolithischen Kultur. Sie geht als Zeichen der Würde des Erwachsenseins den obligatorischen, jedoch erst postpubertär praktizierten „Tatauierungen“ voraus, welche die Haut der Mentawaier am gesamten Körper zieren und die Würde von Mann und Frau symbolisieren. ${ }^{20}$

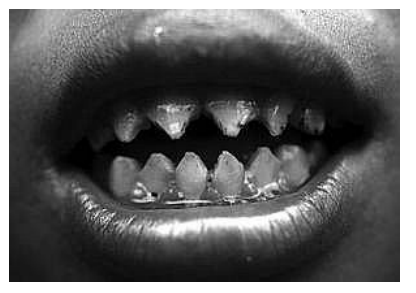

Abb. 3 Mentawaier mit zugespitzten Zähnen

13 Scully/Chen (1994).

14 Aufderheide/Rodríges-Martín (1998).

15 Bethke/Reichart (1999).

16 Armstrong et al. (1995).

17 Schröder (1906), zitiert nach einer Übersicht von Kanner (1928), S. 213.

18 Kanner (1928).

19 Ebd.

20 Zahorka (2001). 


\section{Zahnfeilungen, um Lücken, Kerben oder Zinken zu formen}

Diese Art der Zahnveränderung wird häufig bei Stämmen im Süden Afrikas als Pubertätsritus für beide Geschlechter durchgeführt und oft mit Extraktionen anderer Zähne kombiniert, wie etwa bei den Bantu, bei denen Einkerbungen an den Oberkieferschneidezähnen zusammen mit der Extraktion der vier Unterkieferschneidezähne erfolgen. Diesen Riten liegen vielfach animistischreligiöse Motive zugrunde. So wird die Lücke zwischen den Oberkieferschneidezähnen bei einigen Bantu-Stämmen als „Macht“, „Kraft“ oder „Stärke“ bezeichnet, bei anderen als „Zeichen des Todbringers“. ${ }^{21}$

\section{Extraktion von Zähnen}

Die vollständige Entfernung der oberen mittleren Schneidezähne wurde bei den Frauen der Toraja auf Sulawesi bis weit in das 20. Jahrhundert vorgenommen, während bei den Männern die Kronen in Höhe des Zahnhalses abgebrochen wurden. ${ }^{22}$

\section{Kronenamputation oder horizontales Zurückschleifen der Zähne}

Das horizontale Einkürzen der sechs Frontzähne - insbesondere der Eckzähne - im Oberkiefer wird bis zum heutigen Tage bei hinduistischen Jungen und Mädchen auf Bali als Initiationsritus durchgeführt und stellt gemäß balinesischem Recht eine Voraussetzung für die Ehefähigkeit dar. ${ }^{23}$

\section{Einfärben von Zähnen}

Das Färben der Zähne ist bekannt aus Regionen in Asien, Australien, Afrika sowie in Mittel- und Südamerika. ${ }^{24}$ In vielen Völkern Südost-Asiens gelten natürlich belassene, weiße Zähne als aggressiv, tierisch oder unmenschlich. ${ }^{25}$ In Malaysia schwärzt man sich durch regelmäßiges Kauen von Sirih-Betel auf einfache Weise die Zähne. ${ }^{26}$ In Japan beispielsweise waren schwarze Zähne noch zu Beginn des 20. Jahrhunderts ein Schönheitsmerkmal und daher gerade unter Geishas weit verbreitet. ${ }^{27}$ Verschiedene Indianerstämme in Mittelund Südamerika schwärzen ihre Zähne, während andere rot gefärbte Zähne bevorzugen. Rote Zähne sieht man, wenn auch selten, in Afrika in einer Region von Nigeria sowie in Marokko. Darüber hinaus benutzen manche HinduVölker Rot zum Zähnefärben, da diese Farbe als den Göttern besonders gefällig angesehen wird. ${ }^{28}$

\footnotetext{
21 Kanner (1928).

22 Zahorka (2001).

23 Ebd.

24 Kanner (1928).

25 Zahorka (2001).

26 Zahorka (2001), Kanner (1928).

27 Kanner (1928).

28 Ebd.
} 


\section{Zahndekoration mit Einlagen aus Metall, Gold, Edelsteinen und Diamanten}

In Malaysia und Japan sowie bereits während der Maya-Kultur in Mittelamerika wurden künstlich hergestellte Kavitäten mit individuellen Schmuckeinlagen gefüllt; diese waren nicht nur zur Dekoration bestimmt, sie galten auch als Statussymbole (s. Abb. 4). ${ }^{29}$

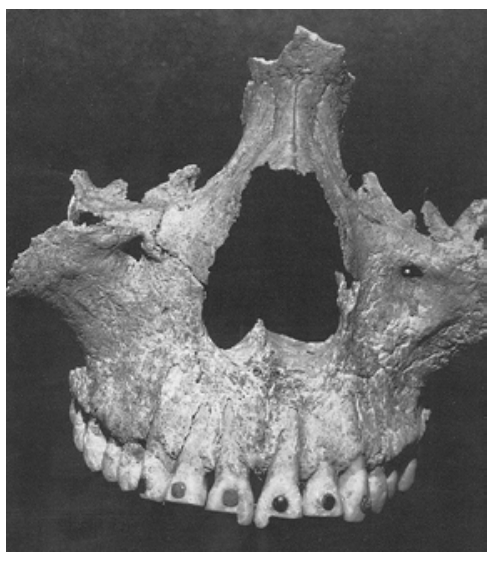

Abb. 4 Oberkieferfragment mit Zahneinlagen und Zahnfeilungen

Auch bei den Batak auf Sumatra kennt man diese Art Zahnschmuck, wobei die Schneidezähne mit runden, die Eckzähne jedoch mit dreieckigen Einlagen verziert werden. Dazu kommen noch auffallende goldene Streifen, die entlang des Zahnfleischsaums angebracht werden. ${ }^{30}$ Die Dayak in Borneo schmücken ihre Schneidezähne mit Kupfer- oder Goldplättchen, welche beim Sprechen unregelmäßig aufblitzen und auf diese Weise eine besondere Wirkung hinterlassen. Von diesem Stamm ist auch das Tragen von Kupfernägeln mit runden Köpfen bekannt, welche durch eine Bohrung in den Schneide- und Eckzähnen geführt werden..$^{31}$

\section{Veränderung der Zahnstellung}

Die Frauen verschiedener Stämme im Senegal haben eine künstliche Prognathie des Oberkiefers entwickelt. Um dieses Ziel zu erreichen, werden bereits im Kindesalter die Oberkieferschneidezähne des Milchgebisses entfernt. Finger und Zunge üben anschließend auf die durchbrechenden bleibenden Zähne einen nach labial gerichteten Druck aus. Dabei werden nicht nur die Schneidezähne in ihrer Position verändert, auch der Alveolarknochen beugt sich dieser Parafunktion, so dass am Ende der Mund eine schnauzenähnliche Form bekommt. ${ }^{32}$

29 Aufderheide/Rodríges-Martín (1998), Kanner (1928). 
Andere Zahnbewegungen werden zum Beispiel bei den Masai durchgeführt. Durch das Setzen von Keilen wird ein künstliches Diastema zwischen den beiden mittleren Schneidezähnen provoziert. ${ }^{33}$

Nach diesem kurzen Rückblick in Geschichte und Kultur soll im Folgenden der Frage nachgegangen werden, welche Körpermodifikationen im Orofazialbereich aktuell in den westlichen Industrienationen bedeutsam sind.

\section{Körpermodifikationen im Orofazial-Bereich: Eine aktuelle Übersicht}

Die WHO schätzt die Prävalenz von Tattoos in der Gesamtbevölkerung der „westlichen Welt“ auf 10\%. ${ }^{34}$ Nach Tenenhaus (1993) waren von 14.109 von der Musterungsbehörde in Paris einberufenen jungen Männern 587, d. h. ca. $4 \%$, tätowiert. 85\% der Tätowierten erhielten ihr Tattoo im Alter von 14 bis 18 Jahren, nur $13 \%$ wurden in jüngeren Jahren tätowiert, noch weniger danach. Copes und Forsyth (1993) geben für die U.S.A. an, dass insgesamt 10\% der Bevölkerung Tattoos tragen, wovon etwa 75 \% Männer und nur rund $25 \%$ Frauen sind.

In einer kürzlich veröffentlichten Studie von Stirn et al. $(2006 \mathrm{a})^{35}$ wird die Verbreitung von Tattoos und Piercings in der bundesdeutschen Bevölkerung im Alter von 14 bis 93 Jahren mit 8,5\% bzw. 6,8\% angegeben. Bei einer Begrenzung auf die Altersgruppe von 14 bis 44 Jahren sind immerhin bereits $15 \%$ der Deutschen tätowiert und $14 \%$ gepierct, und in der Gruppe der 14- bis 24-Jährigen tragen $41 \%$ der weiblichen sowie $27 \%$ der männlichen Subpopulation mindestens ein Piercing oder Tattoo. ${ }^{36}$ Besonders häufig sind Tätowierungen mit 22,4\% bei jungen Männern von 25 bis 34 Jahren, während Mädchen und junge Frauen im Alter von 14 bis 24 Jahren mit $38 \%$ die größte Gruppe der Piercing-Trägerinnen darstellen. Demgegenüber nannte das Magazin Bild der Wissenschaft (02/2001) noch einen Schätzwert von etwa 2 Millionen tätowierter bzw. gepiercter Menschen in Deutschland - also nur rund 2,5\% der Gesamtbevölkerung.

Aussagen über die Häufigkeit von Körpermodifikationen im Orofazialbereich werden hierbei nicht gemacht, jedoch ist davon auszugehen, dass ein beträchtlicher Anteil hierbei auf Zungen- und Lippenpiercings entfällt. Einzelne Studien aus den U.S.A. ${ }^{37}$ und Finnland ${ }^{38}$ geben immerhin bei Studenten eine Prävalenz oraler Piercings von 10,5\% bzw. 3,4\% an. Nach einer Erhebung im Jahr 2006 an mehreren High Schools in Buffalo tragen etwa 10\% der Schüler mindestens ein orales Piercing. ${ }^{39}$

\footnotetext{
33 Ebd.

34 Der Spiegel (2003).

35 Weitere Daten aus derselben Erhebung bei Hinz et al. (2006), Schöne (2006) und Stirn et al. (2006 b).

36 Stirn et al. (2006 b).

37 Mayers et al. (2002).

38 Ventä et al. (2005).

39 Pearose et al. (2006).
} 

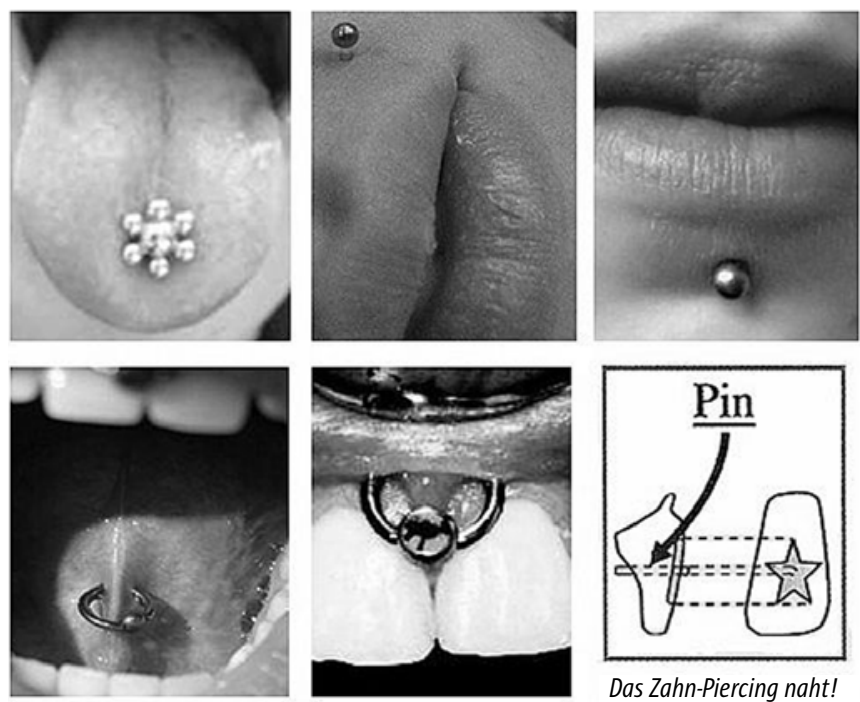

Abb. 5 Zusammenstellung verschiedener orofazialer Piercings

Die Mehrheit der orofazialen Piercings, die besonders bei Jugendlichen eine beachtliche Verbreitung gefunden haben bzw. weiterhin finden, penetrieren neben der Haut in der Regel auch die orale Mucosa sowie Muskeln und Bindegewebe des Gesichts. Je nach Lokalisation lassen sich folgende Piercings unterscheiden:

1. Das Zungenpiercing steckt meistens stabförmig in der Mitte der Zunge, kann aber auch im Randbereich der Zunge liegen und wird dann häufig als Ring getragen.

2. Labretpiercings durchbohren das Gewebe vorwiegend im Bereich der Lippenfurche und bestehen häufig aus einem Stab, der oral einen plattenförmigen Abschluss aufweist und fazial mit einem schraubbaren Schmuckaufsatz versehen wird.

3. Lippenpiercings durchdringen das Gewebe in unmittelbarer Nähe zum Lippenrot und werden überwiegend in Ringform getragen.

4. Piercings der Frenula werden meist durch das Lippen- oder Zungenbändchen gesetzt, in der Regel in Ringform.

5. Das Wangenpiercing durchstößt stabförmig das Wangengewebe.

6. Das Uvulapiercing durchstößt das Zäpfchen des Gaumensegels.

Neben diesen klassischen Piercings erfreut sich das Zungensplitting zunehmender Aufmerksamkeit. Die Spaltung der Zungenspitze kann durch ein Skalpell erfolgen. Alternativ wird die Zunge median gepierct und ein dünner Nylonfaden (Angelschnur) inseriert, der durch regelmäßige Dreh- und Sägebewegung die Zungenspitze innerhalb weniger Wochen durchtrennt. Bei beiden Methoden müssen die Hälften der Zungenspitze regelmäßig auseinander gezogen werden, bis der Heilungsprozess abgeschlossen ist (s. Abb. 6). 


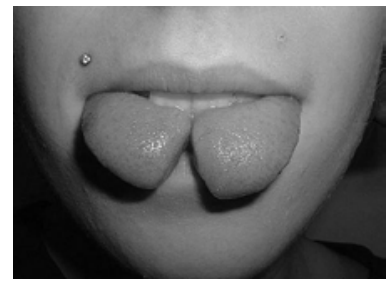

\section{Abb. 6 Zungensplitting}

Tätowierungen im orofazialen Bereich werden in der Regel als „permanent Make-up“ angeboten, um eine dauerhafte Färbung beispielsweise der Lippen oder Wangen zu erreichen oder um einen „Schönheitsfleck“ $z u$ erzeugen. ${ }^{40}$ Darüber hinaus kann natürlich die gesamte Gesichtshaut und die Kopfhaut mit Tätowierungen versehen werden. ${ }^{41}$ Ebenso sind Tattoos auf der Mundschleimhaut möglich (s. Abb. 7).

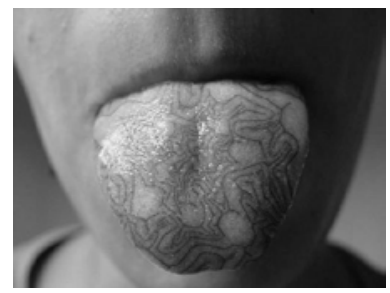

Abb. 7 Zungentattoo

Neben diesen das Weichgewebe betreffenden Körpermodifikationen stellen in der Mundhöhle die Zähne ein beliebtes Objekt für mehr oder weniger invasiven Körperschmuck dar. ${ }^{42}$

$\mathrm{Zu}$ den nicht oder minimal invasiven Zahnschmuckarten zählen diverse Sorten von Schmucksteinen aus Glas, Kristallglas, Zirkon, Diamanten, Brillianten sowie verschiedene andere Edelsteine, welche über Säure-Ätz-Technik und Schmelz-Bonding auf der Zahnoberfläche befestigt werden und unter anderem als „Blizzer“, „Brillance“, „Skyce“, „Twinkles“, „Twizzler“ angeboten werden (s. Abb. 8). Neben Schmucksteinen, welche ungefasst sowie mit verschiedenen Fassungen erhältlich sind, lassen sich auch Goldfolien (z. B. „Dazzler“) in unterschiedlichsten Formen auf den Zähnen fixieren.

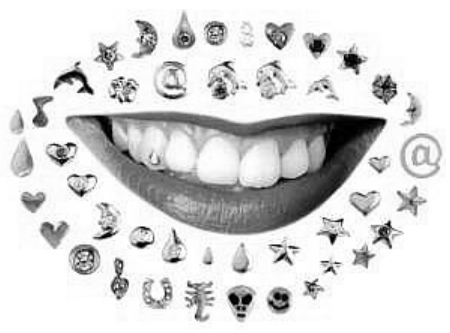

Abb. 8 „Geklebter“ Zahnschmuck

40 Kasten (2006), Chimenos-Küstner et al. (2003).

41 Abbildungen bei Kasten (2006), S. 340.

$42 \operatorname{Groß}(2007 a)$. 
Das Schmelz-Bonding-Verfahren eignet sich darüber hinaus zur Fixierung von individuellen Zahnmalereien, die auf eine mit dem Zahnschmelz verbundene Kunststoffgrundierung aufgebracht und von einer klaren Kunststoffdeckschicht schützend überzogen werden (s. Abb. 9). Denkbar sind auch Kombinationen von Bemalung, Steinen oder Folien.

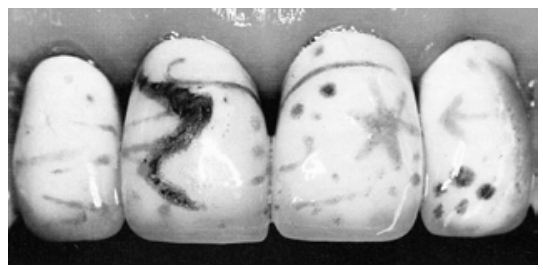

Nicht zu verwechseln mit den kleinen Klebebildchen, welche als „Zahntattoo“ im Handel sind und lediglich wenige Stunden halten, sind „Tattooths“: Diese bestehen aus einem Verankerungselement, auf welches unterschiedliche Schmuckaufsätze nach Wunsch aufgeschoben werden. Um ein „Tattooth“ befestigen zu können, muss der Zahn wie zur Aufnahme einer künstlichen Zahnkrone beschliffen werden. Damit auch das Aussehen eines natürlichen Zahnes simuliert werden kann, gibt es einen speziellen Aufsatz mit der individuellen Zahnfarbe des „Tattooth“-Trägers (s. Abb. 10).

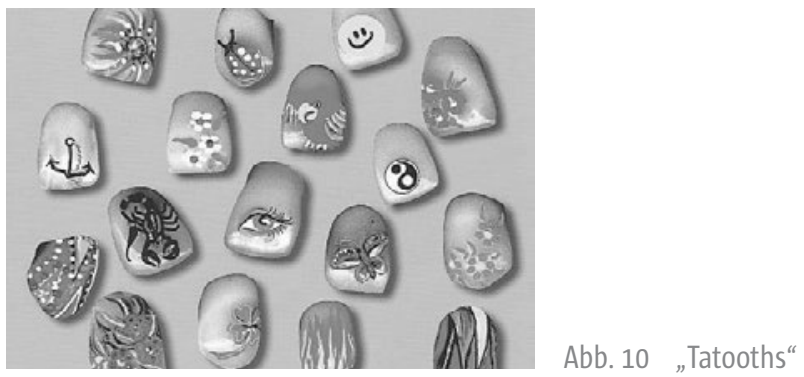

„Grills“ oder „Grillz“ sind nach Abformung der Kiefer individuell gefertigte, über Klammern an den Zähnen verankerte Schmuckspangen, die goldfarbig und häufig mit Strass oder Edelsteinen besetzt sind (s. Abb. 11).

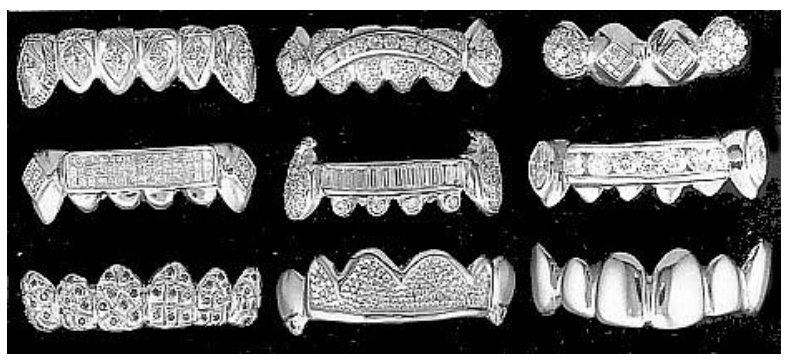

Abb. 11 "Grillz" 
„ZahnCaps“ werden ähnlich wie Kronen auf zuvor beschliffenen bzw. bearbeiteten Kronen getragen. Sie sind ähnlich wie „Grills“ insbesondere in der Rapper-Szene beliebt (s. Abb. 12).

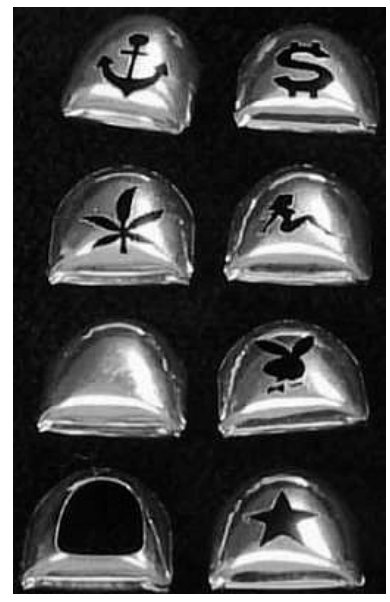

Abb. 12 „ZahnCaps"

Beim „Zahnpiercing“ wird ein Kanal durch den Zahn gebohrt, in welchen ein Schmuck tragender Stecker eingesetzt wird (s. Abb. 5).

\section{Zwischen Selbstbestimmung und Selbstverletzung: Motive und Risiken}

\subsection{Motive}

Was die Motivation von Tätowierten und Piercingträgern betrifft, so sind verschiedene Erklärungsansätze auszumachen. Neben ästhetischen Motiven ist der Wunsch nach Veränderung, nach mehr Individualität, nach Abgrenzung, nach einem besonderen Lebensgefühl, nach dem Spüren des eigenen Körpers und nach einer Erfahrung von Schmerz denkbar. Auch ein gewisser sozialer Druck oder der Wunsch nach Zugehörigkeit zu einer bestimmten sozialen Gruppe sind als mögliche Motive für Piercings und Tattoos anzunehmen.

Ebendiese Motive wurden von Stirn (2003) in einer ersten deutschen Fragebogenerhebung (Durchschnittsalter knapp 29 Jahre) untersucht. Folgende Hauptgründe für die Körpermodifikationen Tattoo oder Piercing wurden hiernach angegeben: An der Spitze standen „Kunst/Schönheit“, dicht gefolgt von „Körpergefühl/Individualität“; an dritter Stelle rangierte der Wunsch nach Veränderung. Das Motiv „Schmerzerfahrung“ besaß als Grund bei den Männern eine ähnlich hohe Relevanz wie „Veränderung“, während bei den Frauen dieser Grund nur sporadisch angeführt wurde. Auch die Motive „Mutbeweis“ und „Aufnahme in eine Gruppe/Gemeinschaft“" wurden nur vereinzelt und ausschließlich von Männern benannt.

Was die Auswirkungen von Piercing oder Tattoo auf das Körperempfinden betrifft, so gaben knapp $54 \%$ an, dass sie ihren Körper nach dem Eingriff 
als „schöner“ empfänden; gut 39\% sahen demgegenüber keine Veränderung. Beim Lebensgefühl stand bei gut $43 \%$ die Individualität im Vordergrund, andere sahen sich auf dem Weg der Selbstverwirklichung (32\%). „Unverändert“ fühlen sich rund $29 \%$, während sich fast $12 \%$,besonders“ fühlen.

Bemerkenswert ist, dass sich fast 81 \% zusätzliche Tattoos oder Piercings zuzulegen wünschten. Ein amerikanischer Verhaltensforscher vergleicht Piercings und Tattoos mit Kartoffelchips: „Einer allein reicht nicht“. ${ }^{43}$ Stirn legt dagegen folgende Interpretation nahe: „Der Wunsch, immer mehr Piercings haben zu wollen, stellt mit an Sicherheit grenzender Wahrscheinlichkeit eine misslungene Identitätssuche dar und kann als Symptom für psychische Konflikte gewertet werden.“44 Diese „Art Sammelleidenschaft“ oder gar „Suchtverhalten " wird von ihr dahingehend interpretiert, dass die erwünschten Effekte wie Steigerung des Selbst- oder Körperbewusstseins oder Erhöhung der Individualität nur passager erhalten bleiben. ${ }^{45}$ Tatsächlich haben Ventä et al. (2005) in ihrer Untersuchung festgestellt, dass sich unter den Studenten mit oralen Piercings ein vom Durchschnittskollektiv abweichendes Suchtverhalten nachweisen ließ. Unter den Trägern von Piercings befanden sich signifikant mehr Raucher und Konsumenten illegaler Drogen als bei Studenten, die keine Piercings trugen. Auch der Anteil an Studierenden mit leichten Depressionen lag bei den Trägern oraler Piercings signifikant höher.

In der Untersuchung von Pearose et al. (2006) in Buffalo wurden Schüler von High Schools bis einschließlich 19 Jahre ebenfalls nach ihren Motiven für orofaziale Piercings befragt. Bei dieser deutlich jüngeren Gruppe standen überraschenderweise andere Motive als in der Studie von Stirn im Vordergrund: $36 \%$ ließen sich „aus Spaß“ piercen, für $27 \%$ stand der „Reiz im Moment der Entscheidung“ im Vordergrund und bei 14\% war das „Anderssein“ das Hauptmotiv. Weitere, nahezu gleich gewichtige Beweggründe waren, dass Geschwister, Eltern oder Freunde Piercings tragen. Damit stellt sich die Frage, ob die Zugehörigkeit zu einer bestimmten Altersgruppe eine bestimmte Motivlage begründet. Zumindest ist auffällig, dass bei den Schülern eine tiefere Motivation fehlt oder die Motivation vielfach unbestimmt ist und die Entscheidung eher spontan getroffen wird.

Kasten (2006) hat bei einer Internetrecherche in Foren und Chatrooms weitere individuelle Beweggründe für „body modifications“ erschlossen: Der eigene Körper wird hiernach nicht länger als unveränderlich hingenommen, er wird umgestaltet und verbessert, um einem - individuellen - Idealbild zu entsprechen. Auffällig ist, dass es nicht bei einer Körpermodifikation bleibt: Die Spaltung der Zunge in Verbindung mit dreißig und mehr Piercings am ganzen Körper sind keine Einzelfälle und haben neben schmückendem auch rituellen Charakter. Bei anderen steht das Hochgefühl des Adrenalinrauschs nach dem Eingriff im Vordergrund. Oder der Körper wird als „unperfekt“ und

43 Schöne (2006).

44 Ebd.

45 Stirn (2004). 
„erbärmlich“ angesehen, während erst durch die Körpermodifikation daraus etwas Schönes geschaffen wird. ${ }^{46}$

Dabei scheint auch die Irreversibilität der Veränderung selbst Teil der Motivation zu sein. Das einmal Geschaffene kann nicht - ohne weiteres - rückgängig gemacht werden, es wird dadurch möglicherweise zum Ausdruck der Suche nach Verbindlichkeit einerseits und zum Zeichen der eigenen Entschlusskraft andererseits.

Das Besondere an der Körpermodifikation liegt darin, dass der eigene Körper selbst zum Objekt der irreversiblen kreativen Veränderung wird - einer Veränderung, die nicht nur als willentliches Zeichen nach außen hin sichtbar ist, sondern zudem eine Wirkung nach innen entfaltet, gleichsam „Unter die Haut" (so der Titel der Ausstellung in Frankfurt am Main) ${ }^{47}$ geht.

Stirn et al. (2006a, b) sowie Hinz et al. (2006) haben in einer weiteren Studie Anhaltspunkte dafür gefunden, dass Menschen mit Körpermodifikationen eine signifikant höhere Risikobereitschaft und einen verstärkten Hang zur Impulsivität zeigen, was die These zulässt, dass durch das Setzen von BodMods ein Ausgleich in Form von neuen und zum Teil sehr intensiven Reizen für den Mangel an körperlichen Herausforderungen in den westlichen Gesellschaften gesucht wird („Sensation-seeking“). Auch wurde festgestellt, dass Träger von Piercings und Tattoos im statistischen Mittel häufiger arbeitslos und seltener in kirchlich-religiöse Gruppen eingebunden sind. Diese Daten könnten die Bedeutung von Körpermodifikationen als Ersatz für traditionelle rituelle Handlungen zur Festigung der gesellschaftlichen Einbindung untermauern. Vielleicht sind sie auch als - neue - Rituale der Integration in Subgruppen zu deuten.

In anderen Studien wurden Exhibitionismus, Kompensation für physische Handicaps, Männlichkeitsinitiation, Bandenmitgliedschaft und Verzierung als mögliche Motive gefunden, ${ }^{48}$ ebenso wurde BodMod als Ausdruck besonders ausgeprägter Extrovertiertheit interpretiert. ${ }^{49}$

Interessant ist ferner die Beobachtung, dass sich viele Heranwachsende ihre Piercings in besonderen Lebensphasen zulegen, etwa um eine spezielle Episode zu „verewigen“ oder deren Ende oder Überwindung zu markieren. Oft geschieht es nach einer Krise, aber auch anlässlich positiver Entwicklungen. Solche „Piercing-Momente“ sind zum Beispiel der bestandene Schulabschluss, das Erreichen der Volljährigkeit oder eine neue Beziehung. ${ }^{50}$

Abschließend ist festzustellen, dass die Motive für die Durchführung von Körpermodifikationen äußerst vielschichtig sind. Insbesondere die am häufigsten angeführten, vordergründig bzw. nach außen wirksamen Aspekte „Schönheit“, „Kunst“, und „Schmuck“ können nicht losgelöst von persönlichkeitswirksamen Aspekten wie „Verbesserung des Körper- oder Lebensgefühls“ sowie „Lust am Reiz“, „Mutprobe und Sensation-Seeking“ betrachtet werden. Auch der Wunsch nach „Individualitätssteigerung“ oder „Identitätsfindung

46 Kasten (2006).

47 Siehe Fußnote 3.

48 Steward (1990).

49 Maio (2006).

50 Schöne (2006). 
oder -stärkung“ spielt eine Rolle, ebenso können die ostentativ demonstrierte Zugehörigkeit zu einer bestimmten sozialen Gruppe und der damit verbundene soziale Druck oder - in der Extremform - der Gruppenzwang eine Motivation darstellen. Damit spielen zwei prima vista diametral entgegen gesetzte Motive - der Wunsch nach einer Verstärkung der Individualität einerseits und der Wunsch, nach außen hin Zusammengehörigkeit zu dokumentieren andererseits - wesentliche Rollen bei der Vornahme von Körperpiercings und Tattoos. Dieser scheinbare Widerspruch ist jedoch aufzulösen, da auch die Zugehörigkeit zu einer bestimmten sozialen Gruppe letztlich wieder dem Wunsch nach Abgrenzung gegenüber anderen sozialen Gruppen bzw. gegenüber führenden oder als dominierend empfundenen Gesellschaftsschichten geschuldet und somit letztlich Ausdruck der eigenen Individualität sein kann.

\section{2 Risiken und Risikobereitschaft}

Wie die angeführten Studien zeigen, sind Körpermodifikationen besonders bei Jugendlichen und jungen Erwachsenen, die eine deutlich erhöhte Risikobereitschaft zeigen, verbreitet. Letztere sind sich der medizinischen Risiken und Folgeschäden, die insbesondere bei orofazialen Piercings nach längerer Tragedauer auftreten, nicht bewusst oder aber nehmen diese leichtfertig in Kauf. Pearose et al. (2006) haben bei ihrer Befragung von Schülern an High Schools die Rückmeldung erhalten, dass 84 \% der Jugendlichen ihr orales Piercing ohne das schriftliche Einverständnis der (zustimmungspflichtigen) Eltern oder Erziehungsberechtigten bekommen hatten. In diesen Fällen ist somit davon auszugehen, dass die Regeln des informed consent, d. h. der aufgeklärten Zustimmung, nicht eingehalten worden sind.

Beim Tätowieren und Setzen von Piercings bestehen zum einen allgemeine Infektionsrisiken, die ihre Ursache in der mangelhaften Umsetzung hygienischer Grundregeln haben. Hier ist insbesondere die Infektionsgefahr durch Viren, Bakterien und Pilze zu nennen. Die möglichen Folgen sind sowohl systemischer als auch lokaler Natur. Als typische lokale Auswirkungen lassen sich Wundinfektionen nennen, welche zu Ödemen, Abszessen oder Phlegmonen führen können. Beispiele für systemische Folgen sind verschiedene Formen von Hepatitis-, Herpes-simplex- und Herpes-zoster- sowie HIV-Infektionen, Endocarditis, Sepsis, Tetanus und Tuberkulose..$^{51}$

Abhängig von den bei Tattoos verwendeten Farbstoffen oder den nach dem Piercen getragenen Schmuckstücken bestehen weitere allgemeine Risiken in der Entwicklung von Hypersensiblitätsreaktionen (Allergien, Urtikaria, Photosensibilisierung bis hin zum anaphylaktischen Schock), Fremdkörperreaktionen wie Granulome, lichenoide Reaktionen sowie maligne Entartungen insbesondere durch krebserregende Stoffe, welche aus den verwendeten Farbstoffen freigesetzt werden..$^{52}$

Da beim Piercen in der Regel mehrere, komplexe Gewebsschichten durchdrungen werden, die gerade in der orofazialen Region von einem dichten Gefäß-

51 Bethke (1999), Groß (2007 a), Kasten (2006), Chimenos-Küstner et al. (2003), Peters (2001).

52 Kasten (2006), Chimenos-Küstner et al. (2003). 
und Nervennetz durchsetzt sind, ist auch bei guten anatomischen Kenntnissen eine Verletzung dieser Strukturen nicht auszuschließen. Verletzungen oder Durchtrennungen von Nerven haben temporäre oder bleibende Ausfallerscheinungen zur Folge: je nach Lokalisation und Art des Nerven kommt es zu Lähmungen, Sensibilitätsstörungen oder -verlust sowie sensorischen Einbußen (z. B. Geschmacksstörungen). Bei Läsionen von Venen oder Arterien treten Blutungen auf, die für den medizinischen Laien oftmals nicht beherrschbar sind oder die zu ausgedehnten Hämatomen führen können. Eine Besonderheit des Orofazialbereichs ist sicherlich auch die Gefahr, dass Schwellungen, ganz unabhängig von ihrer Entstehungsursache, oftmals zur Verlegung der Atemwege führen und daher akut lebensbedrohlich werden können. ${ }^{53}$ Obstruktionen der Atemwege können darüber hinaus auch durch Aspiration von Piercing-Instrumenten oder Schmuckteilen sowie durch profuse Blutungen verursacht werden. ${ }^{54}$

Auf die Wichtigkeit des geeigneten Piercing-Schmucks weisen Peticolas et al. (2000) hin: Gerade bei intraoral gelegenen Piercings sind die Mundbeständigkeit und die biologische Verträglichkeit des verwendeten Materials von besonderer Bedeutung, da sonst Infektionen, allergische und Fremdkörperreaktionen im Bereich des Piercings auftreten können. In diesem Zusammenhang sei auch auf die Untersuchungen von Mortz et al. (2002) an dänischen Schülerinnen und Schülern im Alter von 12 bis 16 Jahren verwiesen. Hier wurde eine statistisch signifikante Abhängigkeit zwischen Nickelallergie und dem Tragen von Ohrringen, jedoch nur für die weibliche Population bestätigt. Bei den männlichen Jugendlichen konnte kein Zusammenhang festgestellt werden. Als Gründe werden eine spätere Exposition, die häufiger anzutreffende Beschränkung auf einen einzelnen Ohrring sowie das Tragen von weniger nickelhaltigem Schmuck bei der männlichen Population diskutiert.

Spezielle Komplikationen nach längerem Tragen von oral situierten Piercings wurden in den letzten Jahren in zahlreichen Fallberichten, klinischen Studien oder Fragebogenaktionen dargestellt. ${ }^{55} \mathrm{Zum}$ einen kommt es insbesondere durch die Ausbildung von Habits wie etwa dem Zungenspiel mit den intraoral gelegenen Metallteilen zur Traumatisierung von Zahnhartsubstanz: Abrasionen, Schmelzabsplitterungen, -risse und -sprünge werden beschrieben, daneben Höcker- und Kronenfrakturen bis hin zum kompletten Zahnverlust. ${ }^{56}$ Aber auch die orale Mucosa und die Gingiva können durch den Piercingschmuck geschädigt werden. Nachweisbar sind Gingivarezessionen im Bereich des Piercings, welche zum Teil zusätzlich erhöhte Sondierungstiefen aufweisen, umgekehrt aber auch gravierende Attachmentverluste ohne auffällige Rezessionen, des Weiteren Gingivitis, Zahnfleischbluten, Druckstellen und die Bildung von Zahnstein am Piercingschmuck. ${ }^{57}$ Eine weitere, öfters be-

53 Bethke/Reichart (1999), Peters (2001), Fehrenbach (1998).

54 Bethke/Reichart (1999), Fehrenbach (1998).

55 Biber (2003), Brooks et al. (2003), Choe et al. (2005), Fehrenbach (1998), López-Jornet et al. (2006), Pearose et al. (2006), Peticolas et al. (2000), Rawal et al. (2004), Soileau (2005), Ventä et al. (2005).

56 Biber (2003), Fehrenbach (1998), López-Jornet et al. (2006), Pearose et al. (2006), Peticolas et al. (2000), Ventä et al. (2005).

57 Biber (2003), Brooks et al. (2003), Choe et al. (2005), Fehrenbach (1998), López-Jornet et al. (2006), Pearose et al. (2006), Peticolas et al. (2000), Rawal et al. (2004), Soileau (2005), Ventä et al. (2005). 
obachtete Folge von intraoral gelegenem Piercingschmuck ist die Zahnwanderung. Hierbei entstehen meistens Diastemata, also Lücken zwischen den Zähnen. Regelmäßig wird auch ein vermehrter Speichelfluss bei Piercingträgern beschrieben..$^{58}$

$\mathrm{Zu}$ den medizinischen Risiken und Unwägbarkeiten gesellen sich, gerade bei den hier betrachteten, meist sichtbaren Körpermodifikationen im Gesichtsbereich, gesellschaftliche Nachteile. Inwieweit Träger von orofazialen Piercings oder Tattoos sozial benachteiligt, stigmatisiert oder pathologisiert werden, soll nachfolgend näher untersucht werden.

\section{Zwischen Akzeptanz und Stigmatisierung: Körpermodifikationen im Orofazialbereich}

Ältere Übersichtsarbeiten wie von Klees-Wambach (1976) belegen, dass Tätowierungen in westlichen Ländern zumindest bis in die jüngere Vergangenheit überwiegend in den unteren sozialen Klassen und im „kriminiellen Milieu“ verbreitet waren. ${ }^{59}$ Doch Tätowierungen und Piercings zieren seit einiger Zeit nicht mehr allein die (Schleim-)Haut bestimmter Naturvölker oder - mit Blick auf den westlichen Kulturkreis - einzelner Gruppen wie Strafgefangener oder Matrosen. Aktuelle Zahlen aus Deutschland, die Aglaja Stirn kürzlich auf einer Tagung in München präsentierte, ${ }^{60}$ zeigen vielmehr einen starken Trend zu Körpermodifikationen in fast allen Bevölkerungsschichten. Ost-West-Unterschiede oder Differenzen zwischen Besserverdienenden und unteren Einkommensgruppen sind diesen Zahlen zufolge kaum auszumachen.

Vor dem Hintergrund dieser Äußerungen könnte man annehmen, dass Körpermodifikationen eine zunehmend gesellschaftliche Akzeptanz erfahren und ihre Träger das Stigma des „Andersseins“ mehr und mehr einbüßen. Diese Schlussfolgerung greift indessen zu kurz: Vielmehr stellte Vandekerckhove (2006) im Rahmen seiner Betrachtungen zur wissenschaftlichen Erforschung des Phänomens Körpermodifikation fest, dass bereits dem Untersuchungsgegenstand Tätowierung - und damit auch dem Untersucher - bislang „ein gewisses Stigma“ anhaftete. ${ }^{61}$ Damit die wissenschaftliche Arbeit aufgrund der mangelnden gesellschaftlichen Wertschätzung dieses Themas nicht in die Bedeutungslosigkeit abgleitet, sieht sich der Forscher genötigt, seine Auseinandersetzung mit dem Phänomen Tätowierung der Gesellschaft gegenüber zu legitimieren. Dies führte bislang zu wissenschaftlichen Herangehensweisen an das Thema Tätowierung, welche die Stigmatisierung des Tätowierens noch verstärkte: Zunächst fand eine Betrachtung aus der anthropologischen Perspektive statt, welche das Tätowierungsphänomen als eine Erscheinungsform fremder,,unzivilisierter "Völker beschrieb und dadurch die Distanz zur westlichen ,Kultur` schon im Ansatz vergrößerte. Die zweite Form des wissen-

58 Bethke/Reichart (1999), Ventä et al. (2005), López-Jornet et al. (2006).

59 Allerdings liegen zur Verbreitung von Tätowierungen in der Mittel- oder Oberschicht keine Vergleichszahlen aus diesen Gesellschaftsgruppen vor.

60 Schöne (2006).

61 Vandekerckhove (2006). 
schaftlichen Interesses war die kriminologische Auseinandersetzung mit dem Thema, welche das Tätowieren zwar nun in der eigenen Gesellschaft, jedoch ausschließlich als Phänomen krimineller oder unterprivilegierter, gleichfalls ,unzivilisierter' Cruppen beleuchtete. Zum Dritten fand die Beschäftigung aus einem psychologisch-psychiatrischen Blickwinkel statt: Sie wurde entweder als pathologisches Verhaltensmuster gedeutet oder wiederum mit dem Konnotat der Devianz belegt oder als Sonderform einer Therapie zumindest in einen Zusammenhang mit Krankheit gestellt, also pathologisiert. Die vierte Annäherung an das Thema Tätowieren geschah aus medizinischer Sicht, welche die Gesundheitsrisiken in den Mittelpunkt der Betrachtung stellte, wodurch indirekt das Tätowieren als Quelle der Gefahr und ,der Tätowierte، als besonders risikofreudig dargestellt wird; auch diese Sichtweise leistet letztlich einer Diskriminierung der Betroffenen Vorschub. Insofern bestätigt die bisherige Auswahl der Perspektiven und Fragestellungen in der wissenschaftlichen Beschäftigung mit dem Thema Tätowierung dessen gesellschaftliche Geringschätzung und Absonderlichkeit. ${ }^{62}$

Doch so sehr die traditionelle Art der Beschäftigung mit dem Thema Tätowierung und Piercing eine Stigmatisierung bzw. Diskriminierung der betroffenen Träger befördert - als einzig zulässige Lesart kann sie schon deshalb nicht gelten, weil sich aktuelle Untersuchungen dem Thema in deutlich vorurteilsärmerer Weise nähern. Die früher häufig angenommene enge Verbindung zwischen (erotisch akzentuierten) Piercings, Sadomasochismus und Fetischismus - die ebenfalls eine Pathologisierung bzw. Psychiatrisierung der Betroffenen nahe legte - ist zu relativieren. Auch die generelle Deutung von intentionalen Körpermodifikationen als „Zeichen einer Autoaggression oder eines selbstverletzenden Verhaltens “ ist Stirn zufolge in Abrede zu stellen. ${ }^{63}$

Gleichwohl gibt es Stimmen, die Body Mod (durchaus pathologisierend) im Sinne einer Persönlichkeitsstörung interpretieren, etwa als Ausdruck einer narzisstischen Haltung, die ein bestimmtes individuelles Schönheitsbild im Blick hat, das unter Umständen gängige Ideale bewusst konterkariert oder die Ausbildung neuer Schönheitsideale befördert ${ }^{64}{ }^{4}$ mmerhin lässt sich für den Orofazialbereich die besondere Bedeutung (durchaus unterschiedlich verstandener) ästhetischer Maßnahmen statistisch belegen: Waren 1999 immerhin 49 \% der Bürger bereit, für dentalkosmetische Maßnahmen Geld auszugeben, so sind es aktuell sogar $63 \%$. Für $86 \%$ der Deutschen hat Zahnästhetik einer Emros-Umfrage zufolge eine große Bedeutung, und $69 \%$ sehen hierin sogar einen sehr hohen Stellenwert - auch dies geht aus einer Umfrage des Jahres 2005 hervor. ${ }^{65}$ Dabei ist vielfach nicht das Erreichen der Norm, sondern gerade die Normabweichung - z. B. unnatürlich helle Zähne durch Dental Bleaching (Zahnbleichen) - das Ziel. Letztlich sind Modifikationen im Orofazialbereich zugleich Ausdruck eines Lebensgefühls, das geprägt ist von Lust, Reiz an Pro-

62 Ebd.

63 Schöne (2006).

64 Stirn (2004).

$65 \operatorname{Groß}(2007 a)$. 
vokation und der Ausformung einer neuen Ästhetik, eines neuen Modetrends und „einer betont körperorientierten Lebensweise“.

Im Übrigen lassen sich auch Argumente geltend machen, die das sozialintegrative Potential von Körpermodifikationen stärker in den Mittelpunkt rücken. ${ }^{66}$ Wenn Körpermodifikationen etwa, wie vielfach beschrieben, das Wohlbefinden und das Selbstwertgefühl steigern, kann dies zugleich positive Rückwirkungen auf die soziale Integration und die soziale Kompetenz der Betroffenen haben. Gleiches gilt für den Fall, dass der Betroffene das Gefühl hat, attraktiver auszusehen, und dies in der Folge in eine besondere Ausstrahlung umzumünzen vermag.

Wenngleich davon auszugehen ist, dass Betroffene in weit geringerem Maße als früher pathologisiert oder direkt gesellschaftlich ausgegrenzt werden, lassen sich doch Situationen ausmachen, in denen BodMod-Anhänger in der Gefahr stehen, gesellschaftliche Nachteile zu erleiden. Anhaltspunkte hierfür liefert etwa Kasten (2006). Er lässt mehrere BodMod-Anhänger konkrete negative Erlebnisse schildern, die sie selbst mit ihrem veränderten äußeren Aspekt in Zusammenhang bringen - so etwa bei der Suche nach einem Arbeitsplatz, im schulischen Umfeld, in der Nachbarschaft oder innerhalb der eigenen Familie. Stirn et al. (2006a) konnten ihrerseits im Rahmen einer Studie bei Männern eine Korrelation zwischen Tattoos und Piercings auf der einen und Arbeitslosigkeit auf der anderen Seite beobachten. Junge Arbeitslose tragen demnach fast doppelt so häufig Piercings wie junge Leute, die eine Anstellung haben oder noch zur Schule gehen. Was hierbei Ursache und was Wirkung ist, muss offen bleiben. Zumindest scheint die Akzeptanz auch vom jeweiligen sozialen Umfeld des Betroffenen abzuhängen.

Dass das Tragen von Piercings oder Tattoos Rückwirkungen auf die gesellschaftliche Akzeptanz und die Vertrauensbildung haben könnte, zeigen auch Untersuchungen zum Einfluss von Gesichtspiercings bei Ärzten: ${ }^{67}$ Während das Tragen von Ohrringen auch bei männlichen Ärzten weitgehend akzeptiert wird, wird Trägern von Nasen- oder Lippenpiercings eine deutlich geringere ärztliche Kompetenz beigemessen. Ähnlich äußern sich die Patienten im Hinblick auf die Vertrauenswürdigkeit von Ärzten mit Ohrringen, Lippen- oder Nasenpiercings. Auch bei den ärztlichen Kollegen stößt das Tragen von Gesichtspiercings oftmals auf Ablehnung: Rund drei Viertel beurteilen Nasenoder Augenbrauenpiercings bei ihren Kollegen als unangebracht und knapp $60 \%$ hätten Probleme, mit einem Kollegen zusammen zu arbeiten, der ein solches Piercing trägt. Der Ohrringschmuck bei männlichen Kollegen wird von rund $39 \%$ als unpassend angesehen und immerhin ein Viertel hätte Schwierigkeiten, mit einem Kollegen oder Medizinstudenten zu arbeiten, der einen Ohrring trägt. Angesichts der geringen Zeit, die einem Arzt für das einzelne Patientengespräch verbleibt, gewinnt die nonverbale Kommunikation, zu der auch das äußerliche Erscheinungsbild des Arztes gehört, zunehmend an Bedeutung. Dies gilt insbesondere für Ärzte und Medizinstudenten, die nur vorübergehend am Behandlungsgeschehen beteiligt sind.

66 Maio (2006).

67 Newman et al. (2005). 
Es lassen sich hingegen ebenfalls Anhaltspunkte für das Entstehen eines Stigmatisierungsphänomens unter umgekehrten Vorzeichen finden: Wer sich kein Tattoo ritzen lassen will oder wer ohne Piercing durchs Leben geht, wird von den Angehörigen bestimmter sozialer Gruppen als „uncool“ bezeichnet oder gar als Außenseiter betrachtet. ${ }^{68}$ Im Zusammenhang mit den Erkenntnissen, dass Körpermodifikationen gerade bei Jugendlichen und jungen Erwachsenen unter 24 Jahren besonders verbreitet sind, ${ }^{69}$ dass unter jungen Menschen mit Körpermodifikationen ein höherer Anteil bereit ist, ein erhöhtes Risiko einzugehen und diese darüber hinaus eher dazu neigen, einem Gruppendruck nachzugeben, ${ }^{70}$ wird das besondere Gefährdungspotenzial deutlich.

Auch aus medizinethischer Sicht fällt die Einschätzung von Körpermodifikationen keineswegs eindeutig aus. Sofern man das Prinzip der Selbstbestimmung zum obersten Prinzip ethischen Handelns in der Medizin erklärt, wird man die Entscheidung zur Körpermodifikation respektieren. Sie trägt der „Patienten“-Autonomie - einem zentralen medizinethischen Prinzip der mittleren Reichweite - Rechnung. Es entspricht einem Grundbedürfnis des Menschen, schön aussehen zu wollen. Es ist nicht der Einzelne, der für sich allein über Schönheit befindet, sondern Schönheit ist eine Norm, eingebettet in eine bestimmte Kultur. Davon abzugrenzen sind subjektive Schönheitsvorstellungen, die höchst unterschiedlich ausfallen und gerade in Tattoos und Piercings ihren Ausdruck finden können. Allerdings sind an die autonome Entscheidung des „Patienten“ Bedingungen zu knüpfen. Der Entscheidung hat eine umfassende Aufklärung vorauszugehen, und der Betroffene muss nach dieser Aufklärung zweifelsfrei seine Zustimmung erklärt haben. Jeder Eingriff in die Integrität des Körpers, wie im betrachteten Fall eine Körpermodifikation, stellt den Tatbestand einer Körperverletzung dar, der gegen das ethische Nicht-Schadens-Prinzip (nonmaleficience) verstößt und allenfalls allein aufgrund der dokumentierten Zustimmung des „Patienten“ legitimiert werden kann. Diese Legitimation gilt jedoch lediglich für einen ärztlichen Eingriff, der seinerseits im Rahmen der grundsätzlichen Aufgabe der Ärztinnen und Ärzte, wie sie in $\mathbb{1} 1$ der (Muster-)Berufsordnung für die deutschen Ärztinnen und Ärzte ${ }^{71}$ wiedergegeben ist, erfolgt. Da das Setzen einer Körpermodifikation kein Eingriff ist, der geeignet wäre, „die Gesundheit zu schützen und wiederherzustellen“ oder „Leiden zu lindern“, ${ }^{72}$ also per se kein ärztlicher Eingriff ist, entpuppt sich der vermeintliche Patient als Kunde und der Arzt mutierte zum Gewerbetreibenden, wenn er denn dürfte. Dem schiebt jedoch $\mathbb{3} 3$ der (Muster-)Berufsordnung gleich zwei Riegel vor. Nach Absatz 1 ist „Ärztinnen und Ärzten neben der Ausübung ihres Berufs [...] die Ausübung einer anderen Tätigkeit untersagt, welche mit den ethischen Grundsätzen des ärztlichen Berufs nicht vereinbar ist“, und Absatz 2 untersagt Ärztinnen und Ärzten, „im Zusammenhang mit der Ausübung ihrer ärztlichen Tätigkeit [...] gewerbliche

68 Ziob (2007).

69 Stirn et al. (2006b).

70 Stirn et al. (2006a, b).

71 www.baek.de/page.asp?his=1.100.1143 [10.04.2007].

72 Ebd. 
Dienstleistungen zu erbringen“. Die Auffassung, dass Piercen keine ärztliche Tätigkeit ist, wird auch von der Bundesärztekammer gestützt. ${ }^{73}$

Derart klare Passagen fehlen hingegen in der Musterberufsordnung der Bundeszahnärztekammer. ${ }^{74}$ So führt $\mathbb{9} 9$ - Praxis - in Absatz 4 aus: „Übt der Zahnarzt neben seiner Tätigkeit als Zahnarzt eine nichtärztliche heilkundliche Tätigkeit aus, so muss die Ausübung sachlich, räumlich und organisatorisch sowie für den Patienten erkennbar von seiner zahnärztlichen Tätigkeit getrennt sein. "Diese Passage muss vor dem Hintergrund einer sich wandelnden juristischen Betrachtungsweise von Körpermodifikationen gesehen werden. Im Jahr 1999 ließ das Verwaltungsgericht Gießen erstmals ein Piercing-Studio schließen, da es das Setzen eines Piercings als Heilkunde definierte und von der Inhaberin eine Erlaubnis nach dem Heilpraktikergesetz forderte. ${ }^{75}$ Auch das Bundesverwaltungsgericht ordnet Tätigkeiten, die ihrer Methode nach der ärztlichen Krankenbehandlung gleichkommen, ärztliche Fachkenntnisse voraussetzen sowie gesundheitliche Schäden verursachen können, ${ }^{76} \mathrm{dem}$ im Heilpraktikergesetz unter $\mathbb{1} 1$ Absatz 2 definierten Begriff der Heilkunde ein. ${ }^{77}$ Dies bedeutet, dass Zahnärzte und Zahnärztinnen, welche die Erlaubnis zur Ausübung der Heilkunde nach dem Heilpraktikergesetz erlangt haben - also befugt sind, nichtärztliche heilkundliche Tätigkeiten im Sinne der Musterberufsordnung der Bundeszahnärztekammer auszuüben -, neben ihrer zahnärztlichen Tätigkeit körpermodifizierende Eingriffe durchführen dürfen, solange sie dies sachlich, räumlich, organisatorisch und für den Patienten nachvollziehbar von der zahnärztlichen Tätigkeit getrennt tun.

Nun ist die Rechtsprechung beileibe nicht eindeutig in der Definition von „Heilkunde“. Während das Verwaltungsgericht Gießen im oben erwähnten Entscheid der Begriff der Heilkunde das Setzen eines Piercings - und im weiter führenden Sinne die Durchführung einer jeden die Integrität des Körpers verletzenden Körpermodifikation - als heilkundliche Maßnahme definiert, ${ }^{78}$

73 Meyer (2001).

74 Vgl. www.bzaek.de/service/oav10/artikel.asp?lnr=295 [10.04.2007]: Nach § 2 - Allgemeine Berufspflichten wird die Zahnärztin/der Zahnarzt lediglich verpflichtet, „sein Wissen und Können in den Dienst der Vorsorge, der Erhaltung und der Wiederherstellung der Gesundheit zu stellen".

75 Verwaltungsgericht Gießen, Beschluss vom 9. Februar 1999 (AZ 8 G 2161/98).

76 Vgl. Bundesverwaltungsgericht, Urteil vom 14.10.1958 (AZ I C 25/56).

77 „Ausübung der Heilkunde im Sinne dieses Gesetzes ist jede berufs- oder gewerbsmäßig vorgenommene Tätigkeit zur Feststellung, Heilung oder Linderung von Krankheiten, Leiden oder Körperschäden bei Menschen, auch wenn sie im Dienste von anderen ausgeübt wird."

78 Das Gericht führt in der Begründung seines Beschlusses aus: „Die inzwischen üblich gewordene Form des Piercens falle nämlich bereits auch ohne die Verabreichung von Lokalanästhetika unter den Begriff der Heilkunde. Darunter fasse $\emptyset 1$ HPG jede Berufstätigkeit oder gewerbsmäßige Tätigkeit zur Feststellung, Heilung oder Linderung von Krankheiten, Leiden oder Körperschäden bei Menschen. Zwar beziehe sich der Normtext dieser Legaldefinition nicht schon auf im weitesten Sinne kosmetisch indizierte chirurgische Eingriffe. Eine an dem bloßen Wortlaut des $\emptyset 1$ Abs. 2 HPG orientierte Auslegung entspreche indessen nicht dem Sinn und Zweck dieses Gesetzes. Nach der vom HPG beabsichtigten Aufhebung der für jedermann auf dem Gebiet der Heilkunde weitgehend möglichen sog. Kurierfreiheit sollte der Kreis derjenigen begrenzt werden, die die Heilkunde auszuüben befugt seien. Unter Berücksichtigung dieser Entstehungsgeschichte werde Heilkundeausübung als eine solche berufsmäßige Tätigkeit angesehen, die besondere ärztliche Fachkenntnisse gebiete. Heilkunde werde daher angenommen, wenn die Tätigkeit nach allgemeiner Auffassung ärztliche oder heilkundliche Fachkenntnisse voraussetze, sei es im Hinblick auf das Ziel, die Art oder die Methode der Tätigkeit selbst, die, ohne Kenntnisse durchgeführt, den Patienten zu schädigen geeignet sei, oder bezüglich der Feststellung, ob im Einzelfall mit der Behandlung 
kommt der Hessische Verwaltungsgerichtshof im selben Fall zu dem Schluss, dass das Setzen eines Piercings nur dann Ausübung der Heilkunde sei, wenn, wie im vorliegenden Fall, ein Arzneimittel zur Lokalanästhesie injiziert wird. ${ }^{79}$ Durch die Rechtsprechung des Hessischen Verwaltungsgerichtshofs werden selbst die Minimalanforderungen, die das Heilpraktikergesetz an die Fähigkeiten und Kenntnisse von heilkundlich Tätigen stellt, ${ }^{80}$ Betreibern von Piercing-Studios, die immerhin chirurgische Eingriffe zur Körpermodifikation durchführen, erlassen.

So nimmt es nicht Wunder, dass insbesondere in ärztlichen, ${ }^{{ }_{11}}$ zahnärztlichen ${ }^{82}$ und auch medizinethischen ${ }^{83}$ Fachkreisen zunehmend die Forderung laut wird, Eingriffe in die körperliche Integrität gehörten in die Hand der medizinischen Professionen, weil diese dafür ausgebildet seien. Auf diese Weise ließen sich Komplikationen beispielsweise durch Unkenntnis der anatomischen Strukturen, durch mangelhaftes operatives Vorgehen oder durch Missachtung der hygienischen Richtlinien für den Kunden reduzieren ${ }^{84}$ Eine

begonnen werden dürfe, und wenn die Behandlung - bei generalisierender und typisierender Betrachtung der in Rede stehenden Tätigkeit - gesundheitliche Schädigungen verursachen könne. Hiervon ausgehend umfasse $\oint 1$ Abs. 2 HPG auch Maßnahmen, die nach seinem Wortlaut keine Ausübung von Heilkunde darstelle, aber mit Rücksicht auf die Gefährlichkeit des Eingriffs oder bezüglich der Frage, inwieweit dieser gefährlich sei, ein besonderes diagnostisches Fachwissen erfordere. Tätigkeiten, die folglich ihrer Methode nach keine Krankenbehandlung im eigentlichen Sinne seien, wegen der Schwere des Eingriffs und der damit verbundenen Folgen aber letztlich der ärztlichen Krankenbehandlung gleichkämen, ärztliche Fachkenntnisse vorausetzten sowie Gesundheitsschäden verursachen könnten, fielen unter den Begriff der Heilkunde. Dementsprechend habe das Bundesverwaltungsgericht sehr früh operative Eingriffe zu rein kosmetischen Zwecken sowie die ebenfalls als kosmetischer Eingriff zu wertende Entfernung von Warzen unter diesen Begriff subsumiert. Nur eine solche, auf die Notwendigkeit ärztlicher oder heilkundlicher Fachkenntnisse abstellende Auslegung werde dem Ziel des HPG gerecht, die Gesundheit der Bevölkerung als ein besonderes wichtiges Gemeinschaftsgut zu schützen. Nach diesen Grundsätzen werde folglich bereits das Piercing ohne Lokalanästhesie von dem Begriff der Heilkunde erfaßt. Hierbei würden nämlich Metallteile in den verschiedensten Formen etwa als Ketten, Ringe, Stecker oder ähnliche Gegenstände nicht nur im gesamten Gesichtsbereich einschließlich der Zunge, sondern auch an unterschiedlichsten Körperstellen angebracht. Diese umfassenden Maßnahmen wolle ersichtlich auch die Studiobetreiberin durchführen, denn es könnten sich die Kunden der Antragstellerin Metallteile an allen Körperteilen anbringen lassen. Mit Recht verweise die Verwaltungsbehörde darauf, daß damit auch hochsensible Nervenstränge nicht nur im Bereich der Zunge, den Augenbrauen, sondern auch zum Beispiel im Genitalbereich tangiert sein könnten. Die damit verbundenen erheblichen Eingriffe in die körperliche Integrität seien geeignet, bei unsachgemäßen Ausführungen zu nachhaltigen Körperschäden zu führen."

79 Hessischer Verwaltungsgerichtshof, Beschluss vom 20.10.2000 (AZ 8 TG 713/99).

80 Beispielhaft seien die schleswig-holsteinischen Richtlinien zur Durchführung des Heilpraktikerrechts angeführt, welche folgende Prüfungsgegenstände umreißen: „Berufs- und Gesetzeskunde einschließlich rechtliche Grenzen der Ausübung der Heilkunde durch Heilpraktikerinnen und Heilpraktiker, Grenzen und Gefahren diagnostischer und therapeutischer Methoden der Heilpraktikerin bzw. des Heilpraktikers, Grundkenntnisse der Anatomie, pathologischen Anatomie, Physiologie und Pathophysiologie, Psychotherapie, Grundkenntnisse in der allgemeinen Krankheitslehre, Erkennung und Unterscheidung von Volkskrankheiten, insbesondere der Stoffwechselkrankheiten, der Herz-Kreislaufkrankheiten, der degenerativen Erkrankungen sowie der übertragbaren Krankheiten, Erkennung und Erstversorgung akuter Notfälle und lebensbedrohender Zustände, Technik der Anamneseerhebung; Methoden der unmittelbaren Krankenuntersuchung (Inspektion, Palpation, Perkussion, Auskultation, Reflexprüfung, Puls- und Blutdruckmessung), Praxishygiene, Desinfektion und Sterilisation, Injektions- und Punktionstechniken, Deutung grundlegender Laborwerte“.

81 Meyer (2001).

$82 \operatorname{Kurz}(2000)$.

83 May/Kohnen (2006), S. 17 f.

84 Vgl. AWMF (2004) für Hygieneanforderungen beim Tätowieren und Piercen. 
berufsrechtliche Regelung in Analogie zur Musterberufsordnung der Bundeszahnärztekammer könnte auf den ersten Blick eine Lösungsmöglichkeit eröffnen: So wäre ein (zahn-)ärztlich ausgebildeter und approbierter Behandler in der Lage, bei einem Kunden - der Begriff Patient verbietet sich in diesem Zusammenhang, da eine Erkrankung nicht vorliegt -, der dies nach eingehender und schonungsloser Aufklärung wünscht, einen körpermodifizierenden Wunscheingriff durchzuführen. Voraussetzung ist, dass er diesen Auftrag dann nicht in seiner Funktion als (Zahn-)Arzt, sondern als Gewerbetreibender ausführt. In seiner Eigenschaft als (Zahn-)Arzt, der seinen Patienten über die Risiken, Komplikationen und Nebenwirkungen eines medizinisch nicht indizierten, körpermodifizierenden Eingriffs aufklärt, ist er dessen Wohl verpflichtet und muss die Durchführung des Eingriffs ablehnen. In der Rolle des gewerblichen Dienstleisters kann er demgegenüber den Eingriff durchführen. Dadurch, dass das Piercing durch einen „Fachmann“ erfolgt, wird das Gesundheitsrisiko des Eingriffs reduziert. Die Rolle des gewerblichen Dienstleisters hat indessen Rückwirkungen auf den Status und die Funktion des (Zahn-) Arztes: Der als (Zahn-)Arzt Approbierte kann nicht zeitweilig seine berufsethischen Grundlagen und Zielsetzungen verlassen und zum gewerblich-chirurgischen Dienstleister ohne Heilauftrag mutieren, ohne den Verlust seiner Glaubwürdigkeit und Authentizität als (Zahn-)Arzt zu riskieren.

Weniger problematisch ist der Umstand, dass körpermodifizierende Maßnahmen nicht für alle Interessenten bezahlbar sind. Derartige Eingriffe stellen keine medizinische Notwendigkeit dar und müssen daher weder allen zugänglich sein noch von der Solidargemeinschaft finanziert werden. Ähnlich ist im Hinblick auf die Kostenfrage bei der Entfernung nicht länger erwünschter Tätowierungen oder Piercings zu argumentieren.

Schwieriger ist dagegen die Beantwortung der Frage, inwieweit Patienten, die sich aufgrund von Komplikationen nach einer Körpermodifikation einer ärztlichen Behandlung unterziehen müssen, an den entstehenden Kosten, welche diejenigen für die Körpermodifikation selbst in der Regel erheblich übersteigen, zu beteiligen sind. Die Neuregelung des $₫ 52 \mathrm{Abs}$. 2 SGB V $V^{85}$ erlegt dies zumindest gesetzlich Krankenversicherten auf, lässt aber die Höhe der finanziellen Eigenbeteiligung offen. So nachvollziehbar das Argument der „Selbstverschuldung“ im Hinblick auf eine Belastung der Solidargemeinschaft auch ist, eine solche Regelung darf nicht dazu führen, dass akut - z. B. an einem Abszess - Erkrankte den notwendigen Schritt einer ärztlichen Therapie hinauszögern und sich hierdurch in ernste Gefahr begeben. Ein weiteres Problem ist in der Ursachenanalyse zu sehen: Wer entscheidet, ob beispielsweise eine Infektionskrankheit wie Hepatitis C, die oftmals erst Jahre nach der eigentlichen Infektion diagnostiziert wird, einem ,,medizinisch nicht indizierten Eingriff" angelastet werden kann? Noch problematischer wird die Selbst-

85 BGBI (2007): $\ 52$ Abs. 2 SGB V: „Haben sich Versicherte eine Krankheit durch eine medizinisch nicht indizierte Maßnahme wie zum Beispiel eine ästhetische Operation, eine Tätowierung oder ein Piercing zugezogen, hat die Krankenkasse die Versicherten in angemessener Höhe an den Kosten zu beteiligen und das Krankengeld für die Dauer dieser Behandlung ganz oder teilweise zu versagen oder zurückzufordern." (Letzte Änderung durch das „Gesetz zur Stärkung des Wettbewerbs in der gesetzlichen Krankenversicherung" vom 26.03.2007, in Kraft getreten am 01.04.2007). 
verschuldensregelung, wenn die Erkrankung erst nach Jahrzehnten als Folge einer Körpermodifikation auftritt, z. B. eine Krebserkrankung durch bei einer Tätowierung eingebrachte, nicht biokompatible Farbstoffe. Ein Ausschluss der Behandlungsmaßnahmen aus dem Leistungsumfang der Krankenkassen wäre für manchen Patienten in der Regel deletär - eine alternative Risikoabsicherung wurde bislang nicht geschaffen, obwohl Lösungsvorschläge wie z. B. eine Pflichtversicherung zur Abdeckung entsprechender Gesundheitsrisiken als fester Bestandteil der Dienstleistung „Körpermodifikation“ bereits in die Diskussion eingebracht wurden. ${ }^{86}$

Desungeachtet tragen die wachsenden Möglichkeiten im Bereich BodMod $\mathrm{zu}$ in der modernen Gesellschaft weit verbreiteten „Machbarkeitsvorstellungen“ bei. Der Mensch lehnt in zunehmendem Maße seine eigenen natürlichen Grenzen ab; er begreift sich selbst nicht mehr als gegeben, sondern nur als gemacht, und dementsprechend verfolgt er Ideen des eigenen „Enhancements" - ein Themengebiet, das allerdings einer gesonderten wissenschaftlichen Analyse bedarf ${ }^{87}$ und überdies nicht mehr unter den Zuständigkeitsbereich der Medizin fällt.

\section{Ausblick}

Schenkt man Vandekerckhove (2006) Glauben, so ist die Gesellschaft der Souverän der Ästhetik. Sie arbeitet dem Aufstieg der Tattoos zum „Kulturgut“ entgegen, indem sie Scham installiert, Geschmack definiert und so vorherrschende Auffassungen verfestigt. Sollte Vandekerckhoves Hypothese zutreffen, zeigt die nivellierende Kraft der Gesellschaft bereits Wirkung, denn einer kürzlich veröffentlichten Umfrage zum Thema Tattoos und Piercings zufolge ist der Wunsch nach Körpermodifikation wieder rückläufig. So stellte das Institut für Demoskopie Allensbach fest, ${ }^{88}$ dass sowohl Tattoos als auch Piercings in der Gesamtbevölkerung ab 16 Jahre zunehmend für unmodern gehalten werden. Zwar halten sich die Bewertungen sowohl für Tattoos (mit $44 \%$,in“ und $41 \%$,out“) als auch für Piercings (mit $43 \%$,in“ und $40 \%$,out“) noch die Waage, doch im Vergleich zum Jahre 2003, als noch $59 \%$ Tattoos und 61\% Piercings als modern bewerteten, ist eine deutliche Veränderung im Trend zu verzeichnen. Eine ähnliche Wende deutet sich auch bei den unter zo-Jährigen an: Letztere halten Körpermodifikationen weiterhin mehrheitlich für zeitgemäß; doch auch hier zeichnet sich eine Trendumkehr ab. So fiel die Einstufung der Tattoos als modern von $70 \%$ (2003) auf aktuell $50 \%$, die der Piercings von $65 \%$ (2003) auf $56 \%$.

Wahrscheinlicher als ein Ende von BodMod ist indessen, dass neue Formen der Körpermodifikation an die Stelle der alten treten. Denn die Geschichte zeigt, dass intentionale Veränderungen des eigenen Körpers zu allen Zeiten und in allen Kulturkreisen eine Rolle spiel $(\mathrm{t})$ en - wenn auch in verschiedenem

\footnotetext{
86 May/Kohnen (2006), S. $18 \mathrm{f}$.

$87 \mathrm{Vgl}$. etwa Groß (2007 b), Ach/Pollmann (2006).

88 Institut für Demoskopie Allensbach (2006).
} 
Ausmaß, in unterschiedlicher Akzentuierung und mit durchaus wechselnden gesellschaftlichen Implikationen und Deutungsmustern.

\section{Literatur}

Ach/Pollmann (2006): Johann S. Ach, Arnd Pollmann (Hrsg.), no body is perfect. Baumaßnahmen am menschlichen Körper - Bioethische und ästhetische Aufrisse, Bielefeld 2006

AWMF (2004): Anforderungen der Hygiene beim Tätowieren und Piercen, in: Arbeitskreis „Krankenhaushygiene" der AWMF (Hrsg.), Hygiene in Klinik und Praxis, Wiesbaden, 3. Aufl., 2004, S. $148 \mathrm{ff.}$

Armstrong et al. (1995): Myrna L. Armstrong, Elaine Ekmark, Barbara Brooks, Body Piercing. Promoting Informed Decision Making, Journal of School Nursing 11 (1995), p. 20-25

Aufderheide/Rodríges-Martín (1998): Arthur C. Aufderheide, Conrado Rodríges-Martín, Soft Tissue Injuries. Mutilation, in: Arthur C. Aufderheide, Conrado Rodríges-Martín (ed.), The Cambridge Encyclopedia of Human Paleopathology, Cambridge 1998, p. 45-50

Bethke/Reichart (1999): Gudrun Bethke, Peter A. Reichart, Risiken des oralen Piercings, Mund-, Kiefer- und Gesichtschirurgie 3 (1999), S. 98-101

Biber (2003): Jay T. Biber, Oral Piercing. The Hole Story, Journal of Northwest Dentistry 82 (2003), p. 13-14, 34

Brooks et al. (2003): John K. Brooks, Kenny A. Hooper, Mark A. Reynolds, Formation of mucogingival defects associated with intraoral and perioral piercing. Case reports. Journal of the American Dental Association 134 (2003), p. 837-843

BGBI (2007): Gesetz zur Stärkung des Wettbewerbs in der gesetzlichen Krankenversicherung, Bundesgesetzblatt (2007), Teil I, Nr. 11, 30.03.2007, S. 378-411

Chimenos-Küstner et al. (2003): Eduardo Chimenos-Küstner, Inés Battle-Travé, Sandra Velásquez-Rengifo et al., Appearance and Culture. Oral Pathology Associated With Certain „Fashions“ (Tattoos, Piercings, etc.), Medicina Oral 8 (2003), p. 197-206

Choe et al. (2005): Jennifer Choe, Khalid Almas, Robert Schoor, Tongue Piercing - Risk Factor to Periodontal Health, The New York State Dental Journal 71 (2005), p. 40-43

Copes/Forsyth (1993): John H. Copes, Craig I. Forsyth, The Tattoo. A Social Psychological Explanation, International Review of Modern Sociology 23 (1993), p. 83-89

Der Spiegel (2003): N. N., Körperschmuck: Autolack in der Haut - Krebsgefahr bei Tattoo-Entfernung? Der Spiegel, 33, 11.08.2003, S. 114

Fehrenbach (1998): Margaret J. Fehrenbach, Tongue Piercing and Potential Oral Complications, Journal of Dental Hygiene 72 (1998), p. 23-25

Groß (2007a): Dominik Groß, Zwischen Wunscherfüllung und Körperverletzung. Die Zahnarztpraxis als Kosmetik- und Wellness-Oase, Vortrag in: Matthias Kettner (Hrsg.), Wunscherfüllende Medizin (Jahrestagung der Akademie für Ethik in der Medizin e. V., Witten-Herdecke 2005), Tagungsbeitrag im Druck, erscheint 2007

Groß (2007 b): Dominik Groß, Neurobionisches und psychopharmakologisches Enhancement, in: Dominik Groß, Sabine Müller (Hrsg.): Sind die Gedanken frei? Die Neurowissenschaften in Geschichte und Gegenwart, Berlin 2007, S. 226-252

Gugutzer (2006): Robert Gugutzer (Hrsg.), body turn. Perspektiven der Soziologie des Körpers und des Sports, Bielefeld 2006

Hinz et al. (2006): Andreas Hinz, Elmar Brähler, B. Brosig et al., Verbreitung von Körperschmuck und Inanspruchnahme von Lifestyle-Medizin in Deutschland, BZgA FORUM Sexualaufklärung und Familienplanung 1 (2006), S. 7-11

Institut für Demoskopie Allensbach (2006): Institut für Demoskopie Allensbach, Tattoos und Piercings. Modetrend: fallend, Umfrage Nr. 7094, Allensbach 2006

Kanner (1928): Leo Kanner, Folklore of the Teeth, New York 1928

Kasten (2006): Erich Kasten, Body-Modification. Psychologische und medizinische Aspekte von Piercing, Tattoo, Selbstverletzung und anderen Körperveränderungen, München 2006

Kissler (2006): Alexander Kissler, Das ist mein Fleisch - Gespaltene Zungen, durchbohrte Glieder, Tattoos als ewige Zeichen: Warum machen die Menschen das? Süddeutsche Zeitung, 05.02.2007, S. 14 
Kurz (2000): Peter Kurz, Piercing im Mund - wenn schon, dann vom Zahnarzt. Die modischen Blender zwischen den Zähnen, Zahnärztliche Mitteilungen 90 (2000), 5, S. 90

López-Jornet et al. (2006): Pia López-Jornet, Christina Navarro-Guardiola, Fabio Camacho-Alonso et al., Oral and Facial Piercings. A Case Series and Review of Literature, International Journal of Dermatology 45 (2006), p. $805-809$

Maio (2006): Giovanni Maio, Ethische Grenzen kosmetischer Maßnahmen in der Zahnheilkunde, Zahnärztliche Mitteilungen 96 (2006), 10, S. 78-83

Mayers et al. (2002): Lester B. Mayers, Daniel A. Judelson, Barry W. Moriarty et al., Prevalence of Body Art (Body Piercing and Tattooing) in University Undergraduates and the Incidence of Medical Complications, Mayo Clinic Proceedings 77 (2002), p. 29-34

May/Kohnen (2006): Arnd T. May, Tanja Kohnen, Körpermodifikationen durch Piercing: Normalität, Subkultur oder Modetrend?, Medizinethische Materialien, Nr. 167, Bochum 2006

Meyer (2001): Rüdiger Meyer, Medizinreport Piercing - Gefährlicher Körperschmuck, Deutsches Ärzteblatt 98 (2001), S. A819 f.

Newman et al. (2005): Alison W. Newman, Seth W. Wright, Keith D. Wrenn et al., Should Physicians Have Facial Piercings? Journal of General Internal Medicine 20 (2005), p. 213-218

Pearose et al. (2006): Maryam M. Pearose, Meghan K. Perinpanayagam, Meelin D. ChinKit-Wells, Trends in Oral Piercing in Buffallo, New York, High Schools, The New York State Dental Journal 72 (2006), p. 30-32

Peters (2001): Siegwart Peters, Piercing. Stellungnahme der DGZMK Version 1.0, Stand 09/2000, Deutsche Zahnärztliche Zeitschrift 56 (2001), S. 132 f.

Peticolas et al. (2000): Troye Peticolas, Terri S. I. Tilliss, Gail N. Cross-Poline, Oral and Perioral Piercing. A Unique Form of Self-Expression, The Journal of Contemporary Dental Practice 1 (2000), 3, p. 1-9

Rawal et al. (2004): Swati Y. Rawal, Lewis J. Claman, John R. Kalmar et al., Traumatic Lesions of the Gingiva: A Case Series, Journal of Periodontology 75 (2004), p. $762-769$

Schöne (2006): Lajos Schöne, Tattoo und Piercing - das neue Lebensgefühl. Körperschmuck ist auf dem Weg zur Normalität, Zahnärztliche Mitteilungen 96 (2006), 21, S. $58 \mathrm{f}$.

Scully/Chen (1994): Crispian Scully, M. Chen, Tongue Piercing (Oral Body Art), British Journal of Oral and Maxillo facial Surgery 32 (1994), p. $37 \mathrm{f}$.

Schröder (1906): Herman Schröder, Die künstliche Deformation des Gebisses. Eine zahnärztlich-ethnologische Studie, Greifswald 1906

Soileau (2005): Kristi M. Soileau, Treatment of a mucogingival defect associated with intraoral piercing, /ournal of the American Dental Association 136 (2005), p. 490-494

Steward (1990): Samuel M. Steward, Bad Boys and Tough Tattoos. A Social History of the Tattoo with Gangs, Sailors and Street-Corner Punks, 1950-1965, New York 1990

Stirn (2004): Aglaja Stirn, Motivationen von Tätowierten und Gepiercten für ihre Körpermodifikationen, Zeitschrift für klinische Psychologie, Psychiatrie und Psychotherapie 52 (2004), S. 43-58

Stirn et al. (2006 a): Aglaja Stirn, Elmar Brähler, Andreas Hinz, Prävalenz, Soziodemografie, mentale Gesundheit und Geschlechtsunterschiede bei Piercing und Tattoo, Psychotherapie - Psychosomatik - Medizinische Psychologie 56 (2006), S. 445-449

Stirn et al. (2006 b): Aglaja Stirn, Andreas Hinz, Elmar Brähler, Prevalence of Tattooing and Body Piercing in Germany and Perception of Health, Mental Disorders, and Sensation Seeking Among Tattooed and Bodypierced Individuals, Journal of Psychosomatic Research 60 (2006), p. 531-534

Tenenhaus (1993): Hervé Tenenhaus, Le tatouage à l'adolescence, Paris 1993

Vandekerckhove (2006): Lieven Vandekerckhove, Tätowierung. Zur Soziogenese von Schönheitsnormen, Frankfurt a. M. 2006

Ventä et al. (2005): Irja Ventä, Ani Lakoma, Sauli Haahtela et al., Oral Piercings Among First-year University Students, Oral Surgery, Oral Medicine, Oral Pathology, Oral Radiology and Endodontology 99 (2005), p. 546-549

Zahorka (2001): Herwig Zahorka, Der Zahn als Kulturobjekt. Dreieckzähne, Zahnschwärzung oder -feilung Traditionen anderer Kulturen, Zahnärztliche Mitteilungen 91 (2001), 16, S. $40 \mathrm{f}$.

Ziob (2007): Brigitte Ziob, Körperinszenierungen - Das veräußerte Selbst, Psyche. Zeitschrift für Psychoanalyse und ihre Anwendungen 61 (2007), S. 125-136 
D Körperwahrnehmung und Identität

\section{Internet}

www.archaeologiemuseum.it/f01_de.html (Südtiroler Archäologiemuseum, Bozen) [07.03.2007]

www.baek.de/page.asp?his=1.100.1143 (Bundesärztekammer, Muster-Berufsordnung für die deutschen Ärztinnen und Ärzte, Stand 24.11.2006) [10.04.2007]

www.bzaek.de/service/oav10/artikel.asp?lnr=295 (Bundeszahnärztekammer, Musterberufsordnung, Stand: 16.02.2005) [10.04.2007]

\section{Bildnachweise}

Abb. 1: aus: http://artcorporel.canalblog.com

Abb. 2: aus: http://perso.orange.fr

Abb. 3: aus: http://jafproject.net

Abb. 4, 5, 9: aus: Sammlung D. Groß, Aachen

Abb. 6: aus: www.pielmag.com

Abb. 7: aus: http://view.stern.de

Abb. 8: aus: www.zahnarztpraxis-pelikan.de

Abb. 10: aus: www.zahnarzt-riedl.de/tatooth.htm

Abb. 11: aus: www.dailywaste.com/images/fronts2.jpg

Abb. 12: aus: http://gangstagold.homestead.com/0000aa.jpg 